\title{
Melioidosis in the Lao People's Democratic Republic
}

\author{
David A.B. Dance 1,2,3,*(i), Manophab Luangraj ${ }^{1}$ (iD), Sayaphet Rattanavong ${ }^{1}$, \\ Noikaseumsy Sithivong ${ }^{4}$, Oulayphone Vongnalaysane ${ }^{5}$, Manivanh Vongsouvath ${ }^{1}$ \\ and Paul N. Newton 1,2,3 \\ 1 Lao-Oxford-Mahosot Hospital-Wellcome Trust Research Unit, Microbiology Laboratory, Mahosot Hospital, \\ Vientiane, Laos; manophab.1@tropmedres.ac (M.L.); Sayaphet@tropmedres.ac (S.R.); \\ Manivanh@tropmedres.ac (M.V.); Paul.Newton@tropmedres.ac (P.N.N.) \\ 2 Centre for Tropical Medicine and Global Health, Nuffield Department of Clinical Medicine, \\ Old Road Campus, University of Oxford, Oxford OX3 7FZ, UK \\ 3 Faculty of Infectious and Tropical Diseases, London School of Hygiene and Tropical Medicine, \\ London WC1E 7HT, UK \\ 4 National Centre for Laboratory and Epidemiology, Vientiane, Laos; noikaseumsy@gmail.com \\ 5 Microbiology Laboratory, Khammouan Provincial Hospital, Thakhek, Laos; bacterialkm@gmail.com \\ * Correspondence: david.d@tropmedres.ac; Tel.: +856-2077475588
}

Received: 29 January 2018; Accepted: 13 February 2018; Published: 19 February 2018

\begin{abstract}
Melioidosis is clearly highly endemic in Laos, although the disease has only been diagnosed regularly in humans (1359 cases) since 1999, and only a single animal case has been microbiologically confirmed. Burkholderia pseudomallei is extensively and abundantly present in soil and surface water in central and southern Laos, but the true distribution of the disease across the country remains to be determined. Surveillance is almost non-existent and diagnostic microbiology services are not yet well established, whilst awareness of melioidosis is low amongst policy-makers, healthcare providers, and the public. It is hoped that this situation will improve over the next decade as the country rapidly develops, especially as this is likely to be accompanied by a further increase in the prevalence of diabetes, meaning that more people in this predominantly agricultural population will be at risk of contracting melioidosis.
\end{abstract}

Keywords: melioidosis; Burkholderia pseudomallei; Laos; Lao PDR

\section{History}

The Lao People's Democratic Republic (Laos) is a land-locked country in Southeast Asia with a population of approximately 6.5 million people, the majority of whom are subsistence farmers, although the country is developing rapidly. Melioidosis was not recognized in Laos until 1999 [1]. Given that Laos is adjacent to highly endemic parts of Thailand, it is likely that the bacterium that causes it has long been present in the environment but that it was not recognized due to a lack of laboratory facilities and awareness amongst healthcare staff. In fact, the detection of Burkholderia pseudomallei in the Lao environment preceded the detection of cases of human melioidosis, although this was not reported until later [2]. An even earlier case was almost certainly acquired in Laos during military action in 1954, although the diagnosis was made in a hospital in Vietnam [3].

\section{Review of Melioidosis Cases and Presence of B. pseudomallei}

\subsection{Human}

Diagnostic microbiology services for human infectious disease have been relatively underdeveloped in Laos compared withmost neighbouring countries such as Thailand, China, and Vietnam. The confirmation of melioidosis as an important public health problem in Laos 
followed the establishment of a research collaboration between the Microbiology Laboratory of Mahosot Hospital, one of the main central referral hospitals in the capital, Vientiane, and the Mahidol-Oxford-Research Unit in Thailand, which had been working on melioidosis in Ubon Ratchathani since 1986. This led to the creation of the Lao-Oxford-Mahosot Hospital-Wellcome Trust Research Unit (LOMWRU), which has supported the provision of diagnostic services for melioidosis, including the use of selective culture media and reagents (Ashdown's agar and broth, latex agglutination and API 20NE) and collated data on culture-positive cases since 1999. Between 2000 and 2004, LOMWRU found B. pseudomallei in 14 patients with bacteraemia, representing 3\% of positive blood cultures [4]. Since then, the number of cases diagnosed has increased each year, reflecting greater awareness of the disease amongst healthcare workers as well as the growing numbers of Lao people with underlying diseases that predispose to melioidosis such as diabetes, which was estimated to have a prevalence of $5.6 \%$ of the Lao population in 2016 [5].

In addition to receiving clinical samples from Mahosot Hospital itself, samples are sent to Mahosot Microbiology Laboratory from other hospitals in and around Vientiane and from other provinces, particularly associated with studies of the aetiology of fever in Luangnamtha, Salavan, and latterly Xiangkhouang provinces. In total, the LOMWRU/Mahosot Microbiology Laboratory has diagnosed 1232 cases of culture-positive melioidosis between 1999 and 2017. The majority came from Vientiane Capital and Vientiane Province, which represent the main catchment areas for Mahosot Hospital; however, since Mahosot Hospital acts as a central referral hospital for parts of Laos and accepts samples from other hospitals for the investigation of possible melioidosis, cases have been seen originating from every province in Laos except Luangnamtha. Details of these cases will be reported elsewhere.

In addition, support was provided by the Centre d'Infectiologie Christophe Mérieux du Laos (CICML) to establish a diagnostic microbiology laboratory in Khammouan Provincial Hospital in Thakhek in 2009, and staff were trained in the recognition of B. pseudomallei and invited to refer any isolates whose identity was uncertain to LOMWRU. Between 2010 and 2017, 79 patients were confirmed with melioidosis, all of whom came from Khammouan Province.

Between 2011 and 2016, the European Union provided $€ 3,000,000$ to support the further development of diagnostic capacity in Laos (https://ec.europa.eu/europeaid/case-studies/ eu-support-epidemiology-and-laboratory-capacity-laos_en). Laboratory staff in Champasak, Savannakhet, Luangphrabang, Oudomxai, and Luangnamtha were trained in the identification of B. pseudomallei and asked to refer suspect isolates to the National Centre for Laboratory and Epidemiology (NCLE) in Vientiane for confirmation. Between 2012 and 2017, this resulted in the identification of a further 48 cases of culture-positive melioidosis.

The data from these three sources have been combined in Table 1, and Figure 1 presents a map showing the distribution of cases and laboratories capable of confirming the diagnosis. An additional 19 Lao patients diagnosed in Thailand [6] and two additional travel-associated cases $[7,8]$ have not been included in the table. Undoubtedly, these figures only represent the 'tip of the iceberg' in Laos, and the distribution of cases across the country is subject to considerable sampling bias. There are still large areas of the country that do not have access to good diagnostic laboratories. Awareness of melioidosis amongst both physicians and laboratory staff remains low, and, even where laboratories do exist, the use of diagnostic microbiology has not yet been fully assimilated into routine clinical practice. The fact that patients usually have to pay for diagnostic tests acts as a further disincentive to investigating the aetiology of infection. 
Table 1. Cases of culture-positive melioidosis diagnosed in Laos ${ }^{1,2}$.

\begin{tabular}{|c|c|c|c|c|c|c|c|c|c|c|c|c|c|c|c|c|c|c|c|c|}
\hline Province & 1999 & 2000 & 2001 & 2002 & 2003 & 2004 & 2005 & 2006 & 2007 & 2008 & 2009 & 2010 & 2011 & 2012 & 2013 & 2014 & 2015 & 2016 & 2017 & TOTAL \\
\hline Attapeu & & & & & & & & & 1 & 1 & & 1 & & & 2 & 3 & & & 1 & 9 \\
\hline Bokeo & & & & & & & & & & & & 1 & & & & & & & & 1 \\
\hline Bolikhamxai $^{1}$ & & & & & 1 & 2 & 3 & 3 & 3 & 3 & 2 & 3 & 2 & 4 & 8 & 8 & 7 & 7 & 12 & 68 \\
\hline Champasak $^{1}$ & & & 1 & 1 & & & & 1 & & 2 & & & & & 1 & 4 & & 3 & 1 & 14 \\
\hline Houaphan & & & & & & & & & & & & 1 & 1 & 1 & & 1 & & & 1 & 5 \\
\hline Khammouan ${ }^{1}$ & & & & & & & 1 & 3 & & 2 & & 10 & 12 & 19 & 12 & 10 & 6 & 8 & 17 & 100 \\
\hline Luangnamtha & & & & & & & & & & & & & & & & & & & & 0 \\
\hline Luangphrabang & & & & & & & & & & & & & & & & 1 & 2 & & & 3 \\
\hline Oudomxai & & & & & & & & & & & & 1 & & & & & & & 1 & 2 \\
\hline Phongsali & & & & & & & & & & & & & & & & 1 & & & & 1 \\
\hline Salavan & & & & & & & & & & 2 & 6 & 4 & 4 & 3 & 3 & 11 & 9 & 4 & 15 & 61 \\
\hline Savannakhet ${ }^{1}$ & & & & & & & & & & & & 1 & 1 & & 1 & 3 & 6 & 1 & 2 & 15 \\
\hline Vientiane Province $^{1}$ & & 1 & 1 & 1 & 1 & 7 & 7 & 9 & 15 & 11 & 19 & 33 & 10 & 13 & 28 & 32 & 33 & 30 & 36 & 287 \\
\hline Vientiane Capital $^{1}$ & & 2 & 6 & 4 & 5 & 25 & 20 & 27 & 51 & 31 & 22 & 58 & 41 & 57 & 66 & 65 & 81 & 108 & 73 & 742 \\
\hline Xaignaburi & & & & & 1 & & & 1 & 1 & 1 & & 2 & & 1 & & 1 & & 4 & 4 & 16 \\
\hline Xekong & & & & & & & & & & 1 & & & & & & & & & & 1 \\
\hline Xaisomboun $^{3}$ & 1 & & & & 1 & & & & 1 & & 1 & 2 & & & 2 & 2 & 3 & 2 & 2 & 17 \\
\hline Xiangkhouang $^{1}$ & & & & & & & & & & & & 1 & & & 1 & 1 & & 1 & 1 & 5 \\
\hline Unknown $^{1}$ & & & & & & 1 & 2 & & & & & & 1 & & & & 2 & 5 & 1 & 12 \\
\hline TOTAL & 1 & 3 & 8 & 6 & 9 & 35 & 33 & 44 & 72 & 54 & 50 & 118 & 72 & 98 & 124 & 144 & 149 & 173 & 167 & 1359 \\
\hline
\end{tabular}

${ }^{1}$ Pooled data from LOMWRU, Thakhek Hospital and NCLE. ${ }^{2}$ Data reflect patient's home village (where known) but not necessarily place of acquisition of melioidosis. ${ }^{3}$ Province established in 2014 


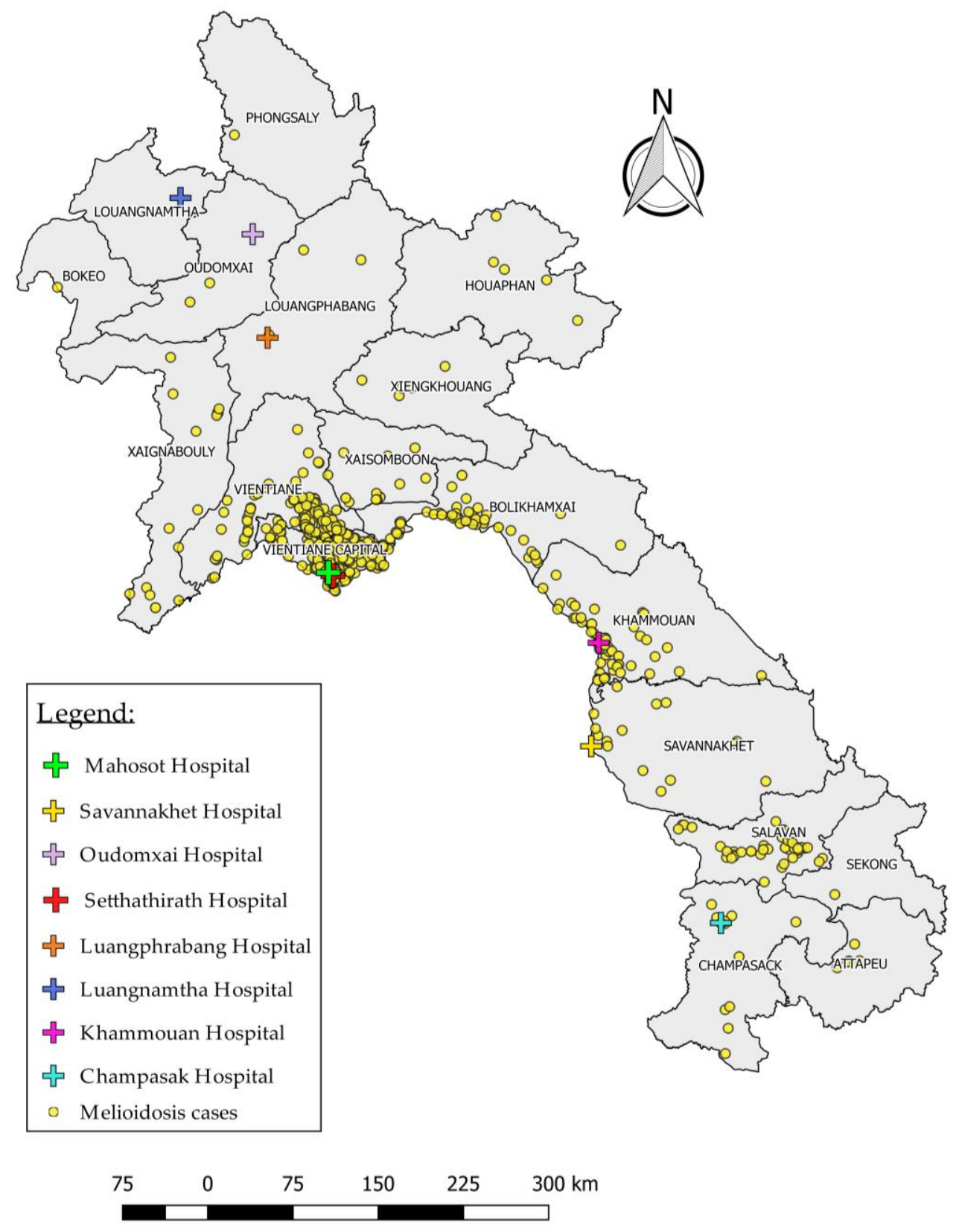

Figure 1. Location of homes of 1310 cases of melioidosis and hospital laboratories capable of making a diagnosis of melioidosis in Laos (data only available for 1310 of 1359 cases).

\subsection{Animal}

Animal meliodosis is undoubtedly present in Laos, although to date only a single case, in a goat, has been confirmed by the LOMWRU laboratory (Newton, P.N.; Vongsouvath, M. (LOMWRU, Vientiane, Lao PDR). Unpublished observations, 2003). Animal surveillance in Laos is limited. Furtherstudies of melioidosis in animals are just beginning to be initiated.

\subsection{Environment}

Several studies have demonstrated that B. pseudomallei is widespread in the environment, at least in central and southern Laos, although the country has not been extensively and systematically sampled to determine the true nationwide distribution of the organism. B. pseudomallei was first isolated in $36 \%$ of 110 soil samples collected in and around Vientiane in 1998 [2]. A subsequent study 
found the organism to be abundantly present in soil in Salavan, but not in a transect from eastern Vientiane to Xiangkhouang [9]. A single isolate from soil in Luangnamtha was reported in this study, although subsequent studies of this isolate have shown that it was actually Burkholderia cepacia that cross-reacted in a B. pseudomallei-specific latex agglutination test (Dance, D.A.B. (LOMWRU, Vientiane, Lao PDR). Unpublished observations, 2011). B. pseudomallei was later confirmed to be present in both soil and surface water in Salavan, including the Sedone river [10]. However, these earlier studies were undertaken using culture alone, which may have underestimated the presence of B. pseudomallei [11]. B. pseudomallei was also found to be readily isolated from a rice paddy in Vientiane about $56 \mathrm{~km}$ from Vientiane's capital [12]. More recently, a nationwide study of the presence of B. pseudomallei in the Mekong and its tributaries in Laos demonstrated its presence in $9 \%$ of the rivers in the dry season and in $57 \%$ of the rivers in the rainy season, mainly in turbid river water with associated B. pseudomallei-positive sediments, and exclusively in the south and centre of the country, suggesting that rivers may be useful in assessing the distribution and aquatic dispersal of B. pseudomallei [13].

\section{Current Recommendations and Availability of Measures against Melioidosis}

\subsection{Surveillance Systems and Reporting}

\subsubsection{Human}

Melioidosis is not statutorily notifiable in Laos and there is no formal surveillance system for human melioidosis. Data are currently only collated by researchers with an interest in the disease as above. Informally, both LOMWRU and the Thakhek laboratories send monthly anonymized reports of episodes of bacteraemia (and other B. pseudomallei isolates in the case of the Thakhek laboratory) to the Ministry of Health and other relevant organisations.

\subsubsection{Animal}

There is no surveillance system for animal melioidosis in Laos.

\subsection{Guidelines}

Melioidosis was included in a manual on diagnosis and treatment of infectious diseases produced (in Lao/English and Lao/French editions) by the Institut de la Francophonie pour la Médecine Tropicale du Laos in 2002 (updated in 2004) that was approved by the Minister of Health. Melioidosis was also included in the 'National Treatment Guideline' (fourth edition) in Lao that was issued in 2012. Both of these were distributed to district and provincial hospitals with the aim of improving the quality of management of infections, including melioidosis, but it is not known how widely available they are and whether they are followed. Neither is available online or outside Laos. The recommendations are also not entirely consistent with current international consensus guidelines on melioidosis treatment [14]. In addition, advice about clinical and epidemiological clues to the diagnosis, which samples to submit, and how to treat patients, have been distributed to users of the Mahosot Microbiology Laboratory through the Mahosot Microbiology Review, an internally distributed newsletter which is available both in English and Lao languages. Diagnostic guidance is contained in a laboratory user manual for the Mahosot laboratory, which is to be issued shortly, and plans are in place to include revised treatment guidelines in updated national antibiotic guidelines that are under development.

\section{Awareness of Melioidosis}

Awareness of the disease amongst health professionals has gradually increased in the area in and around Vientiane since the establishment of LOMWRU but is otherwise patchy, although some clinicians in provinces of relatively high incidence (e.g., Salavan and Savannakhet) regularly consider the diagnosis and manage patients accordingly.The disease is not yet considered a priority by the 
Ministry of Public Health. Although no formal surveys have been conducted, awareness amongst the public is likely to be even lower than that in Thailand [15].

\section{Major Achievements}

Activities relating to melioidosis in Laos are at a very early stage. A case series of the first 1088 patients with melioidosis is in preparation and it is planned that a national workshop, analogous to those that have taken place in neighbouring countries such as Cambodia, will be held in the near future. It is hoped that this will lead to the establishment of a national network of clinicians and researchers with an interest in the disease.

\section{Current and Future Challenges}

Based on the experience at Mahosot Hospital, it is likely that there are still hundreds of people dying of undiagnosed melioidosis in Laos each year, and this is likely to increase as diabetes becomes more common, as it has in neighbouring countries [16]. The biggest challenges facing those who wish to reduce this burden are the relative under-development of diagnostic microbiology services across the country, the lack of a 'culture of culture' amongst clinicians, and a lack of awareness of the disease amongst policy-makers, healthcare providers, and the public. It is hoped that significant progress can be made in these areas over the next decade.

Acknowledgments: The work of LOMWRU is supported by Wellcome (core grant number 106698/Z/14/Z). We are grateful to the doctors, nursing staff, and the staff of the microbiology laboratories of all the hospitals who have provided samples and data, and the Directors of Mahosot Hospital, the Minister of Health and the Director of the Curative Department, Ministry of Health, for their support of these studies.

Author Contributions: D.A.B.D. co-ordinated the review and wrote the first draft. M.L. collated the Mahosot clinical data, populated the table, and prepared the map. N.S. provided NCLE data and O.V. provided the Thakhek data. S.R. participated in most of the environmental studies cited and assisted in the production of the table and map. P.N.N. and M.V. oversaw the routine and research activities relating to melioidosis in LOMWRU and the Mahosot Microbiology Laboratory respectively. All authors reviewed, edited, and approved the final manuscript.

Conflicts of Interest: The authors declare no conflict of interest. The funding sponsors had no role in the design of the study, in the collection, analyses, or interpretation of data, or in the writing of the manuscript.

\section{References}

1. Phetsouvanh, R.; Phongmany, S.; Newton, P.; Mayxay, M.; Ramsay, A.; Wuthiekanun, V.; White, N.J. Melioidosis and Pandora's box in the Lao People's Democratic Republic. Clin. Infect. Dis. 2001, 32, 653-654. [CrossRef] [PubMed]

2. Wuthiekanun, V.; Mayxay, M.; Chierakul, W.; Phetsouvanh, R.; Cheng, A.C.; White, N.J.; Day, N.P.; Peacock, S.J. Detection of Burkholderia pseudomallei in soil within the Lao People's Democratic Republic. J. Clin. Microbiol. 2005, 43, 923-924. [CrossRef] [PubMed]

3. Besseige; Cornet; Trapet; Rigaud; Maury. A propos de deux cas de mélioïdose. Bull. Soc. Pathol. Exot. Filiales 1959, 52, 437-447. [PubMed]

4. Phetsouvanh, R.; Phongmany, S.; Soukaloun, D.; Rasachak, B.; Soukhaseum, V.; Soukhaseum, S.; Frichithavong, K.; Khounnorath, S.; Pengdee, B.; Phiasakha, K.; et al. Causes of community-acquired bacteremia and patterns of antimicrobial resistance in Vientiane, Laos. Am. J. Trop. Med. Hyg. 2006, 75, 978-985. [PubMed]

5. Diabetes country profiles-Lao People's Democratic Republic. Available online: http://www.who.int/ diabetes/country-profiles/en/ (accessed on 10 December 2017).

6. Tanwisaid, K. The melioidosis cases report of Lao People's Democratic Republic patients in Nakhon Phanom Hospital, northeastern Thailand. J. Health Sci. 2008, 17, 1193-1197.

7. Badran, S.; Pedersen, T.I.; Roed, C.; Lunding, S.; Birk, N.; Vestergaard, H.; Roder, B.; Lillelund, H.K.; Kurtzhals, J.A.; Kemp, M.; et al. Imported melioidosis in Danish travellers: A diagnostic challenge. Scand. J. Infect. Dis. 2010, 42, 445-449. [CrossRef] [PubMed] 
8. Tamtami, N.A.; Khamis, F.; Al-Jardani, A. Imported case of melioidosis in Oman: Case report. Oman Med. J. 2017, 32, 62-65. [CrossRef] [PubMed]

9. Rattanavong, S.; Wuthiekanun, V.; Langla, S.; Amornchai, P.; Sirisouk, J.; Phetsouvanh, R.; Moore, C.E.; Peacock, S.J.; Buisson, Y.; Newton, P.N. Randomized soil survey of the distribution of Burkholderia pseudomallei in rice fields in Laos. Appl. Environ. Microbiol. 2011, 77, 532-536. [CrossRef] [PubMed]

10. Vongphayloth, K.; Rattanavong, S.; Moore, C.E.; Phetsouvanh, R.; Wuthiekanun, V.; Sengdouangphachanh, A.; Phouminh, P.; Newton, P.N.; Buisson, Y. Burkholderia pseudomallei detection in surface water in southern Laos using Moore's swabs. Am. J. Trop. Med. Hyg. 2012, 86, 872-877. [CrossRef] [PubMed]

11. Knappik, M.; Dance, D.A.B.; Rattanavong, S.; Pierret, A.; Ribolzi, O.; Davong, V.; Silisouk, J.; Vongsouvath, M.; Newton, P.N.; Dittrich, S. Evaluation of molecular methods to improve the detection of Burkholderia pseudomallei in soil and water samples from Laos. Appl. Environ. Microbiol. 2015, 81, 3722-3727. [CrossRef] [PubMed]

12. Manivanh, L.; Pierret, A.; Rattanavong, S.; Kounnavongsa, O.; Buisson, Y.; Elliott, I.; Maeght, J.L.; Xayyathip, K.; Silisouk, J.; Vongsouvath, M.; et al. Burkholderia pseudomallei in a lowland rice paddy: Seasonal changes and influence of soil depth and physico-chemical properties. Sci. Rep. 2017, 7, 3031. [CrossRef] [PubMed]

13. Zimmermann, R.; Ribolzi, O.; Pierret, A.; Rattanavong, S.; Robinson, M.; Newton, P.N.; Davong, V.; Auda, Y.; Zopfi, J.; Dance, D.A.B. Rivers as transport vehicles and potential sentinels for Burkholderia pseudomallei in Laos. Sci. Rep. 2018, submitted.

14. Lipsitz, R.; Garges, S.; Aurigemma, R.; Baccam, P.; Blaney, D.D.; Cheng, A.C.; Currie, B.J.; Dance, D.; Gee, J.E.; Larsen, J.; et al. Workshop on treatment of and postexposure prophylaxis for Burkholderia pseudomallei and B. mallei infection, 2010. Emerg. Infect. Dis. 2012, 18, e2. [CrossRef] [PubMed]

15. Chansrichavala, P.; Wongsuwan, N.; Suddee, S.; Malasit, M.; Hongsuwan, M.; Wannapinij, P.; Kitphati, R.; Day, N.P.; Michie, S.; Peacock, S.J.; et al. Public awareness of melioidosis in Thailand and potential use of video clips as educational tools. PLoS ONE 2015, 10, e0121311. [CrossRef] [PubMed]

16. Reutrakul, S.; Deerochanawong, C. Diabetes in Thailand: status and policy. Curr. Diabetes Rep. 2016, 16, 28. [CrossRef] [PubMed] 